Ali Pashazadeh*, Michael Friebe

\title{
Transverse dose profile simulation of extruded lines for a 3D printed models for superficial skin cancer therapy
}

\begin{abstract}
The short-range and sharp dose fall-off of beta particles in tissue make them an interesting option for use in the radiation therapy of superficial skin tumors. This can be used to protect bony or other sensitive structures located right beneath the tumor. In a previous study, we studied the feasibility of using 3D printing technology to create $2 \mathrm{D}$ radioactive models for the treatment of skin tumors. In the current study, the Monte Carlo method was used to simulate the transverse dose profile form 3D printed extruded line containing yttrium-90 (Y-90) particles. The time and activity required for treating a superficial skin tumor using these extruded lines were also calculated.
\end{abstract}

Keywords: Beta-emitting isotopes, yttrium-90, superficial skin tumor, 3D printing technology, transverse dose profile.

https://doi.org/10.1515/cdbme-2020-3143

\section{Introduction}

Skin cancer is the most common type of malignancy in humans, with more than 4 million cases diagnosed each year. While there are several types of skin cancer, the most common types are basal cell carcinoma (BCC) and squamous cell carcinoma (SCC), accounting for about $95 \%$ of all skin cancer cases. Patients who are diagnosed with BCC and SCC may be treated by surgery or available alternative options [1]. The most effective treatment substitute for surgery is radiation therapy. This method can also be used as an adjuvant treatment following surgical removal of the skin tumor to kill residual tumor cells. Currently, the established radiation therapy methods applied to skin cancer patients include electron beam therapy, low energy x-ray therapy, and brachytherapy, using a miniature $\mathrm{x}$-ray source or a gamma-emitting isotope such as Iridium-192 [1].

*Corresponding author: Ali Pashazadeh: INKA - Application Driven Research, Otto-von-Guericke University, Magdeburg, Germany, e-mail: ali.pashazadeh@ovgu.de

Michael Friebe: INKA - Application Driven Research, Otto-vonGuericke University, Magdeburg, Germany
Recently, there has been an interest in the use of betaemitting isotopes in treating superficial skin tumors, whose cell killing radiation does not extend very deep in tissue [2-8]. Beta particles emitted from these isotopes, in contrast to $x$-ray and gamma photons, penetrate the tissue usually only up to a maximum of around $10 \mathrm{~mm}$ depth and deposit their dose very locally. This feature can lead to the protection of sensitive structures such as the bone and cartilage near the superficial skin tumor ultimately leading to an improved efficacy-toxicity profile of the treatment. Another potential advantage of the use of beta-emitting isotopes for skin cancer radiotherapy is that there is no further need for the conventional radiation therapy machinery in the hospital to treat these tumors. Instead, a radioisotope provider can supply the hospital with betaemitting isotopes.

In our previous study, we assessed the possibility of 3D printing technology to create planar sources of beta radiation for use in skin cancer therapy [9]. The printed models were intended to be used as radioactive patches on the skin tumor surface. The reason for the application of $3 \mathrm{D}$ printing technology is its capability to conveniently produce customized shapes for patches personalized to the individual tumor size and shape.

In this study hydrogel was used as a base material for 3D printing that could contain printable beta-emitting isotopes. In order to do a proof-of-concept study in an ordinary laboratory, while avoiding the need for a hot lab, the study was performed with non-radioactive particles. A micro-CT scanner was subsequently used to verify the distribution of the particles in the extruded lines.

In the current work, with an aim to push our previous study one step further, we performed a simulation study on the application of the extruded lines of the 3D printer containing beta-emitting isotopes on a skin phantom. The transverse dose profile of one extruded line and several extruded lines printed side by side were calculated. In addition to that, the time and activity needed for treating a superficial skin tumor using a planar beta source consisting of multiple extruded lines were also calculated. 


\section{Method and Material}

As source of radiation in the current study we used the betaemitting isotope Yttrium-90 (Y-90). This radioisotope has been successfully used in several medical applications such as radionuclide therapy of neuroendocrine tumors, radioimmunotherapy of non-Hodgkins lymphoma, and radioembolization of hepatocellular carcinoma [10]. Y-90 has a half-life of about 64 hours and emits beta particles with a maximum energy of $2.28 \mathrm{MeV}$ and an average energy of 0.93 $\mathrm{MeV}$ [10]. The beta spectrum of Y-90 is given in Figure 1. These particles can penetrate up to approximately $11 \mathrm{~mm}$ in tissue. This range makes $\mathrm{Y}-90$ a suitable source for beta radiation therapy of superficial skin tumors.

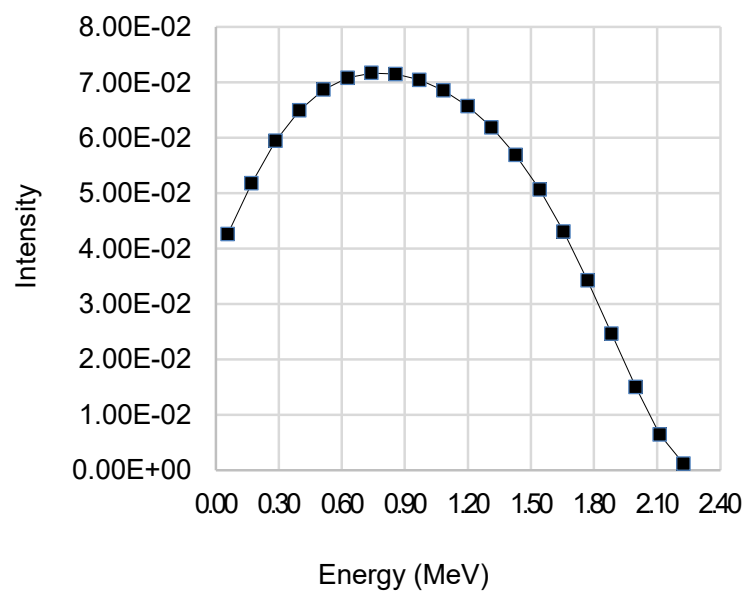

Figure 1 Beta spectrum of Y-90

In order to simulate the radiation dose of 3D-printed extruded lines, the Monte Carlo MCNP4C code was used [11]. The geometry used in the simulation consisted of up to 15 cylinders $(0.7 \mathrm{~mm}$ by $20 \mathrm{~mm})$, and a cuboid $(20 \mathrm{~mm}$ by $20 \mathrm{~mm}$ by $10 \mathrm{~mm}$ ) for the extruded lines and the skin phantom, respectively. The extruded lines were considered to contain uniformly-distributed Y-90 particles. A row of voxels $(0.5 \mathrm{~mm}$ by $0.5 \mathrm{~mm}$ by $0.5 \mathrm{~mm}$ ) was defined right under the surface of the skin phantom and in-plane perpendicular to the axes of cylinder(s). The absorbed dose of beta radiation from the radioactive extruded lines was used to determine the transverse dose profiles.

The code was run first for a geometry consisting of one extruded line placed on a skin phantom. With the transverse dose profile of one line, the code was run for a new geometry consisting of 3 lines laid side by side, with the first line being positioned between the two new lines. There was no separation distance between the consecutive lines. The new geometry was used to assess how the dose profile changes by increasing the number of extruded lines. The study was replicated for 5, 7, 9, 11,13 , and 15 extruded lines laid side by side on the skin phantom, and the transverse dose profile was calculated for each setup.

During the simulation, the material inside the cylinders and the cuboid were considered water, and the number of followed histories in each simulation was set to $10^{7}$. To calculate the absorbed doses, energy tally *F8 was used. This is the tally in the code by which the output of the simulation is set to be energy.

The time and activity needed for treating a typical superficial skin tumor using extruded radioactive lines were also calculated. To this end, a skin tumor with a volume that could be approximated by a cuboid ( $10 \mathrm{~mm}$ by $20 \mathrm{~mm}$ by $3 \mathrm{~mm})$ was used for the simulation. The $2 \mathrm{D}$ area of this tumor can be covered by 15 extruded lines of $0.7-\mathrm{mm}$ diameter and $20-\mathrm{mm}$ length, which were positioned side by side.

The cross-section of the simulation geometry used to calculate the transverse dose profile of one extruded line and the setup for treating a tumor approximated by a cuboid are shown

in

Figure

2.
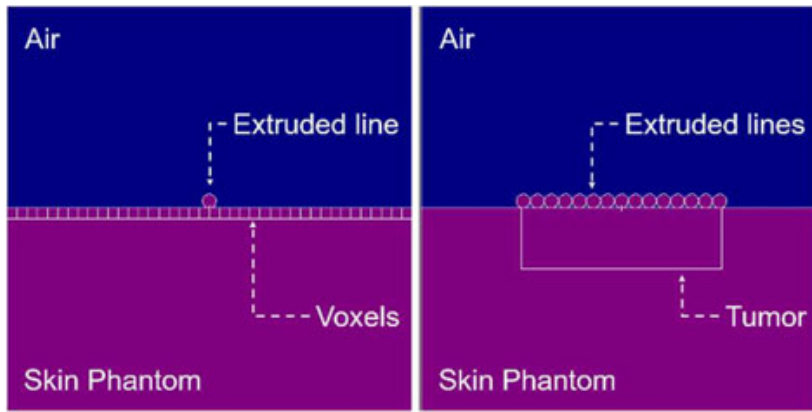

Figure 2 Geometries defined in the MCNP4C to calculate the transverse dose profile of one extruded line (left image), and the setup used to determine the absorbed dose in a tumor volume approximated by a cuboid of $10 \mathrm{~mm}$ by $20 \mathrm{~mm}$ by $3 \mathrm{~mm}$ (right image)

Because *F8 tally scores the deposited energy in $\mathrm{MeV}$, the following equation was applied to convert direct results of the Monte Carlo to Gy/Bq.s, as described in [12]:

$$
\frac{G y}{B q \cdot s}=\left(\frac{\frac{M e V}{g}}{\text { part. }}\right)\left(\frac{1.602 \times 10^{-10} G y}{\frac{M e V}{g}}\right)\left(\frac{\text { ave.part.no. }}{\text { decay }}\right)
$$




\section{Results and discussion}

The transverse dose profiles of the simulated geometries are given in Figure 3. As it was expected, the transverse dose profiles extend to greater off-axis distances by increasing the number of extruded lines laid side by side. As shown in the figure the dose of one extruded line drops to $80 \%$ of the maximum dose at the lateral distance of $<1 \mathrm{~mm}$. For the 15 extruded lines, positioned side by side, the maximum dose drops to $80 \%$ at the lateral distance of about $5 \mathrm{~mm}$. It is also clear from this figure that the transverse dose profiles of all setups have desirable lateral dose uniformity at the first 0.5 $\mathrm{mm}$ layer of the skin phantom.

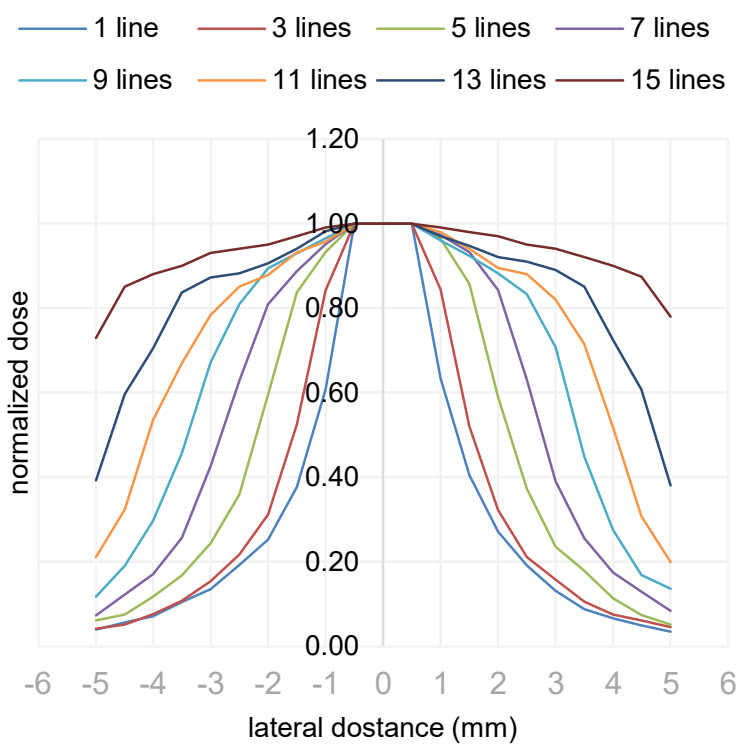

Figure 3 Transverse dose profiles of the extruded lines calculated in the first $05-\mathrm{mm}$ layer of the skin phantom

The simulation result showed that the planar source of Y90 , consisting of 15 extruded lines $(0.7 \mathrm{~mm}$ by $20 \mathrm{~mm})$, printed side by side, can deliver a therapeutic dose of 40 Gy to a cuboid (10 $\mathrm{mm}$ by $20 \mathrm{~mm}$ by $3 \mathrm{~mm}$ ) in about $6 \mathrm{mCi}-\mathrm{h}$. Considering the specific activity of the Y-90 $\left(5.38 \times 10^{9}\right.$ $\mathrm{mCi} /$ gram), about 1.1 nanograms of recently-produced $\mathrm{Y}-90$ will be needed to treat this tumor in one hour. It should be noted that in a real application when there is a delay between the time Y-90 is produced and the time treatment is applied to the tumor, decay correction must be applied in the calculations.

\section{Conclusion}

In this study, the dosimetry study of the concept 3D printing with radioactive material was performed by calculating the transverse dose profile of 3D-printed extruded lines containing beta-emitter Y-90. The result of this simulation study confirmed the symmetry pattern of the dose distribution around the central axis of the extruded lines. This observation, together with the extended dose profiles as a result of increasing the number of extruded lines, shows the possibility of treating superficial skin tumors with different 2D extensions. In a real scenario, the mechanical flexibility of the extruded lines made of hydrogel will be an advantage in treating skin tumors located in surface anatomies that are not flat (e.g., the nose).

\section{References}

[1] A. Pashazadeh, A. Boese, and M. Friebe, "Radiation therapy techniques in the treatment of skin cancer: an overview of the current status and outlook," J Dermatolog Treat, pp. 1-41, Jan 312019.

[2] A. Pashazadeh, R. Landes, A. Boese, M. C. KreissI, M. Klopfleisch, and M. Friebe, "Superficial skin cancer therapy with $\mathrm{Y}$ 90 microspheres: A feasibility study on patch preparation," Skin Res Technol, Jul 232019.

[3] A. Pashazadeh, N. Castro, A. Boese, D. W. Hutmacher, and M. Friebe, "A New 3D Printed Applicator with Radioactive Gel for Conformal Brachytherapy of Superficial Skin Tumor," presented at the 41st Annual International Conference of the IEEE Engineering in Medicine \& Biology Society, Berlin, Germany, 2019. [4] A. Pashazadeh et al., "Conceptual design of a personalized radiation therapy patch for skin cancer," Current Directions in Biomedical Engineering, vol. 4, pp. 607-610, 2018.

[5] A. Pashazadeh, M. Robatjazi, N. J. Castro, and M. Friebe, "A multiwell applicator for conformal brachytherapy of superficial skin tumors: A simulation study," Skin Res Technol, Dec 272019.

[6] Y. L. Chung, J. D. Lee, D. Bang, J. B. Lee, K. B. Park, and M. G. Lee, "Treatment of Bowen's disease with a specially designed radioactive skin patch," Eur J Nucl Med, vol. 27, no. 7, pp. 842-6, Jul 2000.

[7] A. Mukherjee, U. Pandey, H. D. Sarma, M. R. Pillai, and M. Venkatesh, "Preparation and evaluation of $90 \mathrm{Y}$ skin patches for therapy of superficial tumours in mice," Nucl Med Commun, vol. 23, no. 3, pp. 243-7, Mar 2002. 
Ali Pashazadeh et al., Feasibility of 3D printing with radioisotopes for use in superficial skin cancer therapy: A simulation study of the transverse dose profile of the extruded lines $-\mathbf{4}$

[8] M. J. Salgueiro et al., "Design and bioevaluation of a 32Ppatch for brachytherapy of skin diseases," Appl Radiat Isot, vol. 66, no. 3, pp. 303-9, Mar 2008.

[9] A. Pashazadeh et al., "Feasibility of 3D printing for customized radiotherapeutic models to be used in superficial skin cancer therapy," vol. 1, no. 1, 2019.

[10] V. Goffredo, A. Paradiso, G. Ranieri, and C. D. Gadaleta, "Yttrium-90 (90Y) in the principal radionuclide therapies: an efficacy correlation between peptide receptor radionuclide therapy, radioimmunotherapy and transarterial radioembolization therapy.
Ten years of experience (1999-2009)," Crit Rev Oncol Hematol, vol. 80, no. 3, pp. 393-410, Dec 2011.

[11] J. F. J. V. C. Briesmeister, LA-13709-M, Los Alamos National Laboratory, "MCNPTM-A general Monte Carlo N-particle transport code," vol. 2, 2000.

[12] A. Afzalifar, M. Bashi, A. A. Mowlavi, M. R. Fornasier, and H. R. Baghani, "Monte Carlo based determination of dose distribution for some patch sources employed for radionuclide skin therapy," Australas Phys Eng Sci Med, Aug 282018. 